\title{
El papel de las redes en la innovación social: la ciudad de Valencia ${ }^{1}$
}

\author{
J. Salom (a), M. Pitarch (b), R. Mesa (c), E.Margaix (d)
}

(a) Instituto Interuniversitario de Desarrollo Local, Universitat de Valencia, Julia.Salom@uv.es (b) Instituto Interuniversitario de Desarrollo Local, Universitat de Valencia, Maria.Pitarch@uv.es (c) Instituto Interuniversitario de Desarrollo Local, Universitat de Valencia, Rafael.Mesa@uv.es

(d) Instituto Interuniversitario de Desarrollo Local, Universitat de Valencia, Elena.Margaix@uv.es

\section{Resumen}

En los últimos años, el uso del concepto de innovación social como estrategia de desarrollo se ha generalizado tanto en el ámbito académico como entre los tomadores de decisiones. La conformación de una red de actores que interactúe y coopere es un factor importante para la creación y difusión de estas innovaciones. Mediante técnicas de análisis cualitativo y de redes sociales, se determina y evalúa la red de actores y la colaboración entre ellos en la ciudad de Valencia (España), con el fin de definir sus aspectos positivos y negativos y elaborar recomendaciones políticas.

Palabras clave: Geografía Económica, Redes, Innovación social, Economía Social, Valencia

\section{INTRODUCCIÓN: REDES E INNOVACIÓN SOCIAL}

En el año 2011, la Unión Europea publicó "Empowering people, driving change: Social innovation in the European Union", un análisis sobre la definición y objetivos de la innovación social desarrollado por el BEPA (Bureau of European Policy Advisers) cuya finalidad era señalar la relevancia de la innovación social en cualquier ámbito de acción pública a nivel europeo. Hasta entonces, la idea de innovación social existía, al menos de manera parcial o fragmentada, en algunos Programas e Iniciativas de la UE. Pero es a partir de este momento cuando pasa a formar claramente parte de los dos documentos principales que marcan la agenda de los siguientes años: la Estrategia 2020 y el Acta del Mercado Único. Además, y de forma más concreta, la Comisión Europea financia un Programa Europeo para el Empleo y la Innovación Social (EaSI), que se define en su página web como un "instrumento financiero a escala de la UE, cuyo objeto es promover un elevado nivel de empleo de calidad y sostenible, que aporte una protección social correcta, combata la exclusión social y la pobreza y mejore las condiciones de trabajo" ${ }^{2}$. Entre sus objetivos finales destacan, entre otros, promover la equidad, combatir las discriminaciones, combatir el desempleo y luchar contra la pobreza y la exclusión. Estos objetivos ya nos dan una idea sobre la definición de un término, innovación social, que se encuentra muy relacionado con nociones como solución y transformación y que se ha generalizado a partir de la crisis económica, financiera e institucional que ha sufrido Europa desde 2008. La innovación social es un concepto complejo por cuanto sus límites son difusos y dependen, en muchas ocasiones, de la concepción política.

De cualquier forma, no es éste el lugar de debatir sobre su definición, por lo que, para concretar seguiremos la tesis fruto de trabajos previos (Salom et al., 2017), en los que proponemos que la innovación social sea entendida como una serie de iniciativas capaces de ir modificando las rígidas estructuras económicas y sociales para conseguir un cambio en las relaciones entre agentes, instituciones y personas.

En este sentido, entendemos innovación social como una idea, o una nueva forma de aplicar una vieja idea, que aparece cuando la sociedad re-examina cómo se pueden hacerse las cosas, es novedosa en el contexto en que se inserta (no es necesario que sea una innovación absoluta), resuelve retos sociales, culturales, económicos

\footnotetext{
${ }^{1}$ Esta comunicación se ha elaborado en el marco del proyecto "Sostenibilidad social, conectividad global y economía creativa como estrategias de desarrollo en el Área metropolitana de Valencia" (CSO2016-74888-C4-1-R), financiado por la Agencia Estatal de Investigación (AEI) y al Fondo Europeo de Desarrollo Regional (FEDER) dentro del Programa Estatal de Investigación, Desarrollo e Innovación Orientada a los Retos de la Sociedad, en el marco del Plan Estatal de Investigación Científica y Técnica y de Innovación 2013-2016, convocatoria de 2016.

${ }^{2}$ http://ec.europa.eu/social/main.jsp?catld=1081\&langld=es
} 
y/o ambientales, puede ser llevada a cabo por el sector público, privado, o tercer sector, puede ser endógena (surgir de las personas que la necesitan) o exógena (de personas que quieren ayudar), se orienta al beneficio común de la población, y se realiza contando con la participación de la comunidad y los beneficiarios, que se transforman en actores de su propio desarrollo. Todo ello fortalece el sentimiento de ciudadanía y es capaz de crear nuevos vínculos de colaboración con fuerte impacto a nivel local (Salom et al., p. 48).

Pero además de tener una definición, es fundamental, como dice Mulgan et al. (2007), conocer por qué es importante, cómo puede acelerarse, qué actores están implicados, qué resistencias existen, y cuáles son las fases de desarrollo de una innovación social, desde su creación hasta su difusión. En este sentido, la literatura económica de los últimos veinte años ha venido subrayando la importancia de las redes de actores en los procesos de innovación y, en particular, en el impacto territorial de estos procesos. Ello se repite en distintos enfoques de análisis que coinciden en la idea de que la existencia de interrelaciones resulta decisiva para la competitividad de los territorios (Camagni, 1991; Cooke y Morgan, 1993; Morgan, 1997; Bathelt et al. 2004, Méndez, 2007, etc.).

El papel de las redes ha sido resaltado en particular por su impacto en los procesos de aprendizaje, a través de los vínculos de cooperación, confianza e interdependencia que favorecen los intercambios de tecnologías, la provisión de servicios especializados, la circulación de flujos de información formal y sobre todo informal, y, en último término, la creación de procesos de aprendizaje colectivo en entornos territoriales innovadores (Maillat, 1996) o regiones inteligentes (Morgan, 1997).

Según Klein et al. (2015, p. 235), lo específico de la innovación social es la creación o adopción de nuevas formas de hacer, nuevas coordinaciones y nuevas regulaciones, tales como la gobernanza distribuida, la coproducción de servicios, o las actividades de co-construcción de políticas públicas, así como el carácter plural de la economía, tanto en cuanto al modo de propiedad como a la capacidad de hibridación de diversos recursos y, por tanto, la pluralidad de actores. Ya que la producción y la asignación de los recursos no está coordinada sólo por mecanismos de mercado, sino también por sistemas de intercambio basados sobre la reciprocidad y la asociación, el entorno institucional democrático es de la mayor importancia a fin de que sirva de catalizador para la cooperación y la interacción de las redes, con actores responsables de la coordinación y de las acciones compartidas entre ellos (Dufays y Huybrechts, 2014, Moulaert y Nussbaumer, 2015, Slimane y Lamine, 2017).

Sistemas de innovación social de éxito, como el de Quebec, muestran algunas peculiaridades en cuanto a las configuraciones institucionales y en las modalidades de coordinación y de gobernanza que es interesante replicar o al menos tener en cuenta. Entre estas peculiaridades destacan la participación de una pluralidad de actores y la hibridación de diversas formas de gobernanza (Klein et al., 2015, p. 193). En este sentido, el denominado "ecosistema de innovación quebequés" (Levesque, 2011) está compuesto por agentes que cumplen de forma interrelacionada cinco funciones principales: financiación, formación, investigación, servicios y gobernanza, con algunas características destacables:

- En relación con la financiación, existen fuentes de financiación suficientes y diversificadas, provenientes tanto de autofinanciación como de los poderes públicos y de fondos específicos

- En cuanto a la formación, existen universidades, fundaciones y/o agrupaciones empresariales que proporcionan una formación profesional transversal a los diversos sectores de actividad económica.

- La investigación es desarrollada por equipos mixtos de universidad y empresas sociales; a partir de fondos regionales y de las propias universidades; y con una metodología de investigación-acción participativa

- Existe una oferta específica de servicios a las empresas y organizaciones sociales, preferentemente a escala local.

- Y, finalmente, se ha articulado una gobernanza partenarial más horizontal que vertical, que ha desarrollado esquemas de co-construcción de políticas en todas las fases, y con una perspectiva transversal a los diversos sectores de actividad

Las redes de actores resultan, pues, básicas para el desarrollo y mantenimiento de iniciativas de innovación social. De hecho, la colaboración, la creación de redes, se encuentra en la base misma de la idiosincrasia de este tipo de innovación, ya que el aislamiento no es deseable ni genera resultados de éxito. Resulta evidente que el grado de desarrollo de las redes sociales es producto del propio desarrollo de la innovación social y su enraizamiento en el territorio. A continuación analizamos el caso de Valencia, en la que desde hace menos de una década se han puesto en marcha interesantes iniciativas de innovación social como respuesta a problemas de diversa índole, sobre todo sociales, y que han generado una serie de redes incipientes de colaboración que hacen prever la consolidación de este fenómeno en la ciudad. 


\section{EL CASO DE LA INNOVACIÓN SOCIAL EN LA CIUDAD DE VALENCIA (ESPAÑA)}

En Valencia, la política desarrollada desde finales de los 90 del siglo pasado, basada en la construcción de edificios singulares y la promoción de grandes eventos, ha generado un espacio urbano fuertemente fragmentado y crecientemente dualizado (Torres y García, 2013). En este contexto, la identificación de las iniciativas de innovación social en esta ciudad resulta de interés para profundizar en las condiciones sociales y territoriales que explican su surgimiento, evolución y desarrollo, con lo que ello supone de cambio y mejora de la calidad de vida en esta ciudad.

La identificación de las iniciativas de innovación social en Valencia se ha realizado a partir del estudio de casos (Salom et al., 2017). Las 79 iniciativas identificadas hasta la fecha se han agrupado en siete categorías ordenadas teniendo en cuenta el objeto de la innovación de cada una de ellas, siendo la categoría "Dinámicas sociales e inclusión" la que agrupa un mayor número, que supone el $24 \%$ del total, seguida de las iniciativas reunidas en la categoría de "Redes de intercambio y economía colaborativa" con un 19\%. Un poco por debajo se encuentra "Ecología urbana y consumo" que supone un 15\%, y "Urbanismo y patrimonio" con un 13\%. Las categorías con un porcentaje menor son las "Estructuras de apoyo al emprendedor" que suponen un 11\% por ciento, "Educación" con un 10\% y "Fomento del empleo y orientación profesional", con un 9\% de las iniciativas identificadas.

Estas iniciativas innovadoras se concentran geográficamente en un número limitado de barrios y distritos de la ciudad, avalando la hipótesis de que su surgimiento está estrechamente relacionado con las condiciones sociodemográficas, económicas y territoriales del entorno en que aparecen. Se trata de procesos sociales complejos en los que intervienen distintas variables, que sólo es posible aprehender mediante un pormenorizado análisis cualitativo, todavía en desarrollo. Con los primeros resultados es posible avanzar algunas hipótesis, como el hecho de que la existencia de iniciativas parece estar relacionada con el nivel de renta de la población, con la vinculación a movimientos ciudadanos y con la tradición asociativa de los barrios.

Sin embargo, el tema que aquí nos ocupa es la creación de redes de colaboración de diverso tipo y entre agentes tanto públicos como privados y del tercer sector. Analizamos, a continuación, los primeros resultados a este respecto.

\subsection{Hipótesis y metodología}

Nuestra hipótesis de trabajo es que la existencia de un sistema regional o local de innovación, articulado por agentes que ejercen distintas funciones, interactúan y cooperan, es un factor importante en el desarrollo de un territorio innovador. Por tanto, hemos intentado verificar si en la ciudad de Valencia se ha desarrollado o se está desarrollando un sistema de este tipo que favorezca y apoye las iniciativas de innovación social.

Para ello, y partiendo de la información recopilada a partir de encuestas a las entidades y empresas que han desarrollado iniciativas de innovación social, hemos aplicado metodologías de análisis de redes sociales ("network analysis") que nos han permitido dibujar la red de actores que participan y colaboran en las iniciativas de innovación social de la ciudad, identificando la existencia de relaciones de colaboración explícitas tanto de las entidades entre sí como con otros agentes económicos, sociales y comunitarios (administraciones, empresas, bancos, asociaciones, etc.). La información de partida es la proporcionada en la red por las entidades que han realizado iniciativas de innovación social, completada posteriormente mediante encuestas telefónicas o personales a los responsables de la iniciativa. Estos han respondido a la pregunta de con qué entidades y organismos habían establecido o establecían frecuentemente relaciones de colaboración (participación conjunta en proyectos, participación en redes más o menos formalizadas, cofinanciación, etc.). A partir de esta información se ha podido representar el sociograma de relaciones de cooperación (figura 1), y medir el papel de intermediación que juegan los distintos agentes. Para la representación gráfica y los cálculos se han utilizado los programas informáticos Gephi y Ucinet.

\subsection{El análisis de redes sociales. Primeros resultados}

El análisis de redes sociales constituye una aproximación teórica que explica los procesos sociales y el comportamiento de los individuos basándose no sólo en sus atributos, sino considerándolos también un resultado de su participación en relaciones sociales estructuradas. Este análisis considera que la estructura de la red de relaciones entre actores, y el posicionamiento del actor en la red son aspectos con importantes consecuencias en el comportamiento, percepción y actitud de las unidades individuales y del conjunto del sistema. Desde esta perspectiva, 


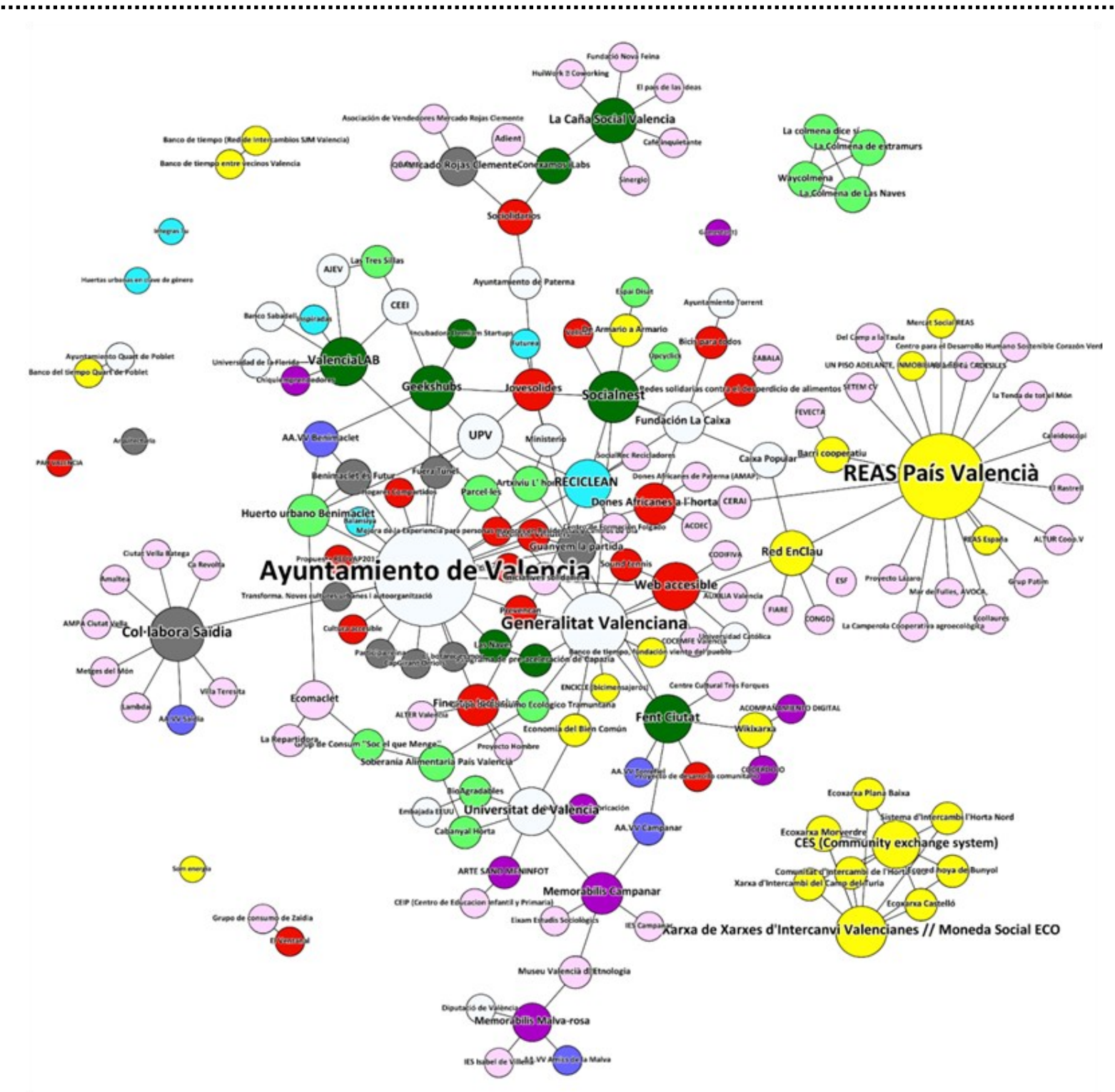

Figura 1 - Red de relaciones de cooperación de las entidades implicadas en la innovación social en la ciudad de Valencia. El tamaño del nodo es proporcional al número de conexiones directas (grado). Fuente: Elaboración propia.

las relaciones entre actores son canales de transferencia y distribución de recursos, materiales o inmateriales; la densidad de los agrupamientos y los tipos de relaciones estructuran el flujo de recursos; y las posiciones estructurales de los agentes, tales como las de gatekeeper (que controla el acceso a la red) o broker (que enlaza dos redes) son características relevantes que permiten obtener poder y mayor acceso a los recursos (Rodríguez, 1995).

Los resultados de nuestro análisis han sido significativos en tres aspectos: la identificación del número y tipo de agentes participantes en la red; la densidad y estructura de la red de relaciones; y la posición de centralidad o marginación de los agentes o tipos de agentes.

Como agentes presentes en el sistema se han considerado no sólo las propias empresas, organismos y entidades que han desarrollado proyectos de innovación, sino también todos los actores, privados, públicos o del tercer sector que mantienen relaciones de colaboración con ellos en el ámbito de la innovación social. Se han diferenciado dos niveles territoriales: el local (área metropolitana) y el supralocal (región, país, otros países). En la red están presentes los siguientes tipos de agentes:

1.- Ámbito local:

1.1. -Empresas y entidades que han desarrollado proyectos de innovación social 
1.2.- Administraciones local y regional

1.3.- Asociaciones y Organizaciones empresariales

1.4.- Universidades y centros de formación

1.5.- Asociaciones sociales y comunitarias

2.- Ámbito supralocal:

2.1.- Administraciones estatales

2.2.- Redes de asociaciones y empresas

2.3.- Entidades financieras

Se han identificado 166 nodos (agentes) con 189 conexiones (relaciones), lo que supone un índice de densidad del 1,4\%, lo que caracteriza la red como muy poco densa, prácticamente incipiente, y todavía con iniciativas desconectadas entre sí, y con el resto de los agentes. El sistema de relaciones se establece preferentemente en el ámbito local, aunque también existen otros agentes, principalmente entidades financieras, asociaciones y organizaciones empresariales, de ámbito regional o estatal.

Para identificar los actores más importantes en la red, se han utilizado dos tipos de índices que tiene en cuenta, respectivamente, la centralidad local (grado o degree) y global (betweenness o posición intermediaria) de los agentes individuales, por un lado, y de los distintos tipos de agentes, por otro (Freeman, 1979). Se supone que a mayor centralidad mayor es la implicación del actor en la red de relaciones, y por tanto más importante su papel.

Un nodo es localmente central si tiene un alto número de conexiones con los nodos de su entorno inmediato; es decir, que un actor es localmente central en términos de conexiones directas con sus vecinos. La figura 2 representa la red de relaciones de colaboración, mostrando los nodos (agentes) en tamaño proporcional al número de conexiones directas o grado. Como puede verse, la mayor centralidad local la ostentan la administración local (Ayuntamiento de Valencia) y, en segundo lugar, estructuras de colaboración de segundo grado como la Red de Economía Alternativa y Solidaria del País Valenciano (que a su vez se incardina en REAS a nivel nacional), y la Xarxa de Xarxes d'Intercamvi Valencianes). A un nivel más modesto, pero también con un importante número de conexiones, se encuentra el Gobierno Regional (Generalitat Valenciana).

El segundo índice calculado mide la centralidad global de los nodos. Un nodo es globalmente central si ocupa una posición estratégicamente significativa en la estructura global de la red. La centralidad global se expresa en términos de distancia entre varios puntos. Betweeness es una medida de centralidad que mide el grado en que un nodo está situado entre los otros nodos de la red. Este índice nos indica en qué medida un actor puede hacer de "broker" (intermediario) o "gatekeeper" (portero), con el consiguiente poder y potencial de control, ya que el actor que está en medio puede controlar interacciones entre otros actores de la red y, por tanto, tiene poder sobre ciertos caminos de interacción.

La tabla 1 y la figura 2 muestran respectivamente los 10 actores mejor posicionados, y la media de este índice para los distintos tipos de actores de la red. Como puede verse (tabla 1), las administraciones local y regional, en particular el Ayuntamiento de Valencia, ocupan un papel central en esta red, seguidos por algunos centros de servicios a las empresas (Fent Ciutat, SocialNest), las universidades, y algunas asociaciones y redes temáticas como REAS País Valenciá, red Enclau, ONGs como Jovesolides, etc. Los agentes externos mejor posicionados son las entidades financieras.

Si entramos en el análisis detallado de las entidades que desarrollan las iniciativas (fig. 2), es posible encontrar diferencias significativas entre las entidades según el tipo de proyectos que han desarrollado. Así, las que han desarrollado proyectos relacionados con las dinámicas sociales y de inclusión, probablemente con una mayor trayectoria de intervención en este ámbito, se encuentran inmersas en una red de relaciones más densa, mientras que las redes de intercambio y economía colaborativa se ubican en el otro extremo, mostrando una dinámica relativamente autónoma.

Si analizamos esta red a la luz de las consideraciones realizadas más arriba, es posible destacar algunos aspectos tanto positivos como negativos. Entre los aspectos positivos cabe señalar la presencia de todos los agentes clave, el papel central de las administraciones públicas y de los centros de servicios a las empresas, y la importante participación de los agentes sociales y comunitarios. Sin embargo, como aspectos negativos hay que señalar la aún escasa densidad de la red y la desigual integración de las iniciativas según su tipo, así como el insuficiente desarrollo de algunas funciones clave como la formación e investigación, y, sobre todo, la ausencia de un sistema de gobernanza transversal y cooperativo que articule el conjunto del sistema. 
Tabla 1 - Actores con mayor centralidad global (índice Beetweenness). Fuente: Elaboración propia.

\begin{tabular}{|c|c|c|c|}
\hline RANGO & NOMBRE & TIPO & BETWEENNESS \\
\hline 1 & Ayuntamiento de Valencia & Institución & 4.030 \\
\hline 2 & Generalitat Valenciana & Redes de Intercambio y economía colaborativa & 3.853 \\
\hline 3 & REAS País Valencià & Dinámicas sociales e inclusión & 2.218 \\
\hline 4 & Jovesolides & Redes de Intercambio y economía colaborativa & 1.916 \\
\hline 5 & Red EnClau & Institución & 1.771 \\
\hline 6 & Ayuntamiento de Paterna & Institución & 1.536 \\
\hline 7 & Universitat de València & Dinámicas sociales e inclusión & 1.493 \\
\hline 8 & Sociolidarios & Fent Ciutat & Estructuras de apoyo al emprendedor social \\
\hline 10 & Socialnest & 1.430 & 1.237 \\
\hline
\end{tabular}

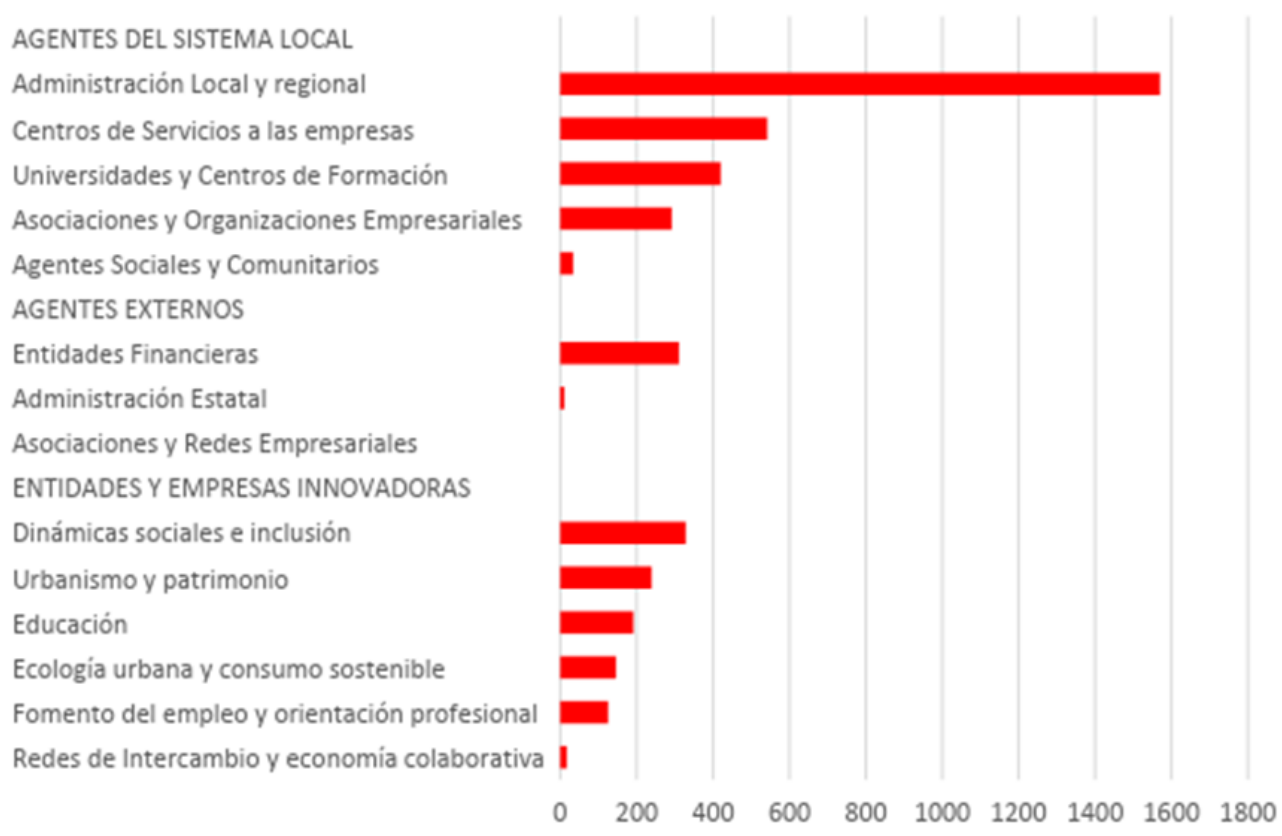

Figura 2. Índice medio de centralidad (Betweenness) de los tipos de actores del sistema local de innovación de la ciudad de Valencia. Fuente: Elaboración propia.

\section{CONCLUSIÓN}

El análisis que hemos realizado nos ha permitido identificar en la ciudad de Valencia el surgimiento reciente de una serie de iniciativas de innovación social en distintos ámbitos temáticos (dinámicas sociales e inclusión, redes de intercambio y economía colaborativa, ecología urbana y consumo, urbanismo y patrimonio, educación, empleo, etc.) cuyo origen está estrechamente relacionado con las condiciones sociodemográficas, económicas y territoriales del entorno en que aparecen, y en particular con el capital social acumulado a lo largo de una trayectoria de movilizaciones sociales y respuestas cooperativas. Los actores que han desarrollado estas iniciativas se relacionan entre sí en un incipiente y aún poco denso sistema de relaciones de colaboración en el que participan también las administraciones local y regional, las universidades y centros de formación, los centros de servicios a las empresas, y algunas entidades financieras, asociaciones y organizaciones empresariales, así como otros agentes sociales y comunitarios tipo asociaciones de vecinos y ONGs. Este sistema muestra un importante déficit en algunas funciones estratégicas, tales como la investigación y la formación, y carece de un marco de gobernanza transversal y participativa, por lo que consideramos que la potenciación de este incipiente ecosistema puede ser una de las políticas locales clave de apoyo a la innovación social. 
El compromiso de la Administración para promover plataformas abiertas hacia la innovación (Laboratorios de Innovación Social) puede satisfacer las necesidades y responsabilidades sociales existentes. El liderazgo público puede mejorar el ecosistema de Innovación Social del territorio, desarrollando mecanismos de financiación de I+D+i social, facilitando la incubación y aceleración de iniciativas, la formación en competencias emprendedoras y haciendo explícitas las demandas de innovación social dentro de la administración. Además, la interacción entre diferentes acciones que surgen a nivel micro genera el ambiente relacional adecuado para crear un sistema reticular articulado entre proyectos o ideas que comparten un objetivo de índole similar. El éxito de estas buenas prácticas reside en su capacidad para hacerlas evidentes y compartirlas en el marco de otras experiencias a diferentes escalas. De este modo, los procesos de Innovación Social en la lógica global de las redes contribuyen a la expansión y a la ampliación de su impacto.

En definitiva, se trata de desarrollar un marco político que conduzca a una transformación socio-cultural que proporcione una mayor resiliencia de los procesos de innovación social, contribuyendo a difundirlos y extenderlos por el territorio.

\section{BIBLIOGRAFÍA}

BATHELT, H., MALMBERG, A. Y MASKELL, P. (2004). Clusters and knowledge: local buzz, global pipelines and the process of knowledge creation, Progress in Human Geography, 28 (1), 31-56.

CAMAGNI, R. (ed.) (1991). Innovation networks. Spatial Perspectives. GREMI, Belhaven Press, Londres

COOKE, PH. Y MORGAN, K. (1993). The network Paradigm. New departures in corporate and regional development. Society and Space, 11, 543-564

DUFAYS, F. Y HUYBRECHTS, B. (2014). Connecting the dots for social value: A review on social networks and social entrepreneurship, Journal of Social Entrepreneurship, 5 (2), 214-237.

FREEMAN, L. (1979): Centrality in Social Networks: I. Conceptual classification, Social Networks, 40, pp. 35-41 1982

KLEIN, J.L.; FONTAN, J.M; HARRISSON, D. Y LÉVESQUE . B. (2015). L'innovation sociale au Québec : un système d'innovation fondé sur la concertation, en Klein. J.-L,; Laville, J.-L, y Moulaert, F. (dirs.) : L'innovation sociale, Érès, Toulouse, 193-218

LÉVESQUE, B. (2011): Innovations sociales et pouvoirs publics: vers un système québécois d'innovtion dédié à l'économie sociale et solidaire. Quelques éléments de problématique, les Cahiers du CRISES, Collection Études Théoriques, ET1106.

MENDEZ, R. (2015): Redes de colaboración y economía alternativa para la resiliencia urbana: una agenda de investigación, Biblio3W. Revista bibliográfica de Geografía y Ciencias Sociales, v XX, 1.139, en red: http://www.ub.edu/geocrit/b3w-1139.pdf

MORGAN, K. (1997). The learning region: institutions, innovation and regional renewal, Regional Studies 31, 491-503

MOULAERT, F. Y NUSSBAUMER, J. (2015). Pour repenser l'innovation: vers un système régional d'innovation sociale, en Klein, J.-L., Laville, J.IL., Moulaert, F. : L'innovation sociale, Eds. Érès, Toulouse. 81-113

MOULAERT, F., MACCALLUM, D., \& HILLIER, J. (2013). Social innovation: intuition, precept, concept. The International Handbook on Social Innovation: collective action, social learning and transdisciplinary research, 13-39.

MOULAERT, F., MARTINELLI, F., GONZÁLEZ, S., Y SWYNGEDOUW, E. (2007). Introduction: Social innovation and governance in European cities urban development between path dependency and radical innovation. European Urban and Regional Studies, 14 (3), 195-209.

MOULAERT, F.; MARTINELLI, F.; SWYNGEDOUW, E. Y GONZÁLEZ, S. (2010): Can Neighbourhoods Save the City? London and New York, Routledge

MULGAN, G., TUCKER, S., ALI, R., Y SANDERS, B. (2007). Social Innovation. What it is, why it matters and how it can be accelerated. Skoll Centre for Social Entrepreneurship. Oxford Said Business School. The Young Foundation.

RODRÍGUEZ, J.A. (1995): Análisis estructural y de redes, Cuadernos Metodológicos, Centros de Investigaciones Sociológicas, Madrid

SALOM J., PITARCH, M.D. Y SALES, A. (2017): "Innovación social: estrategias urbanas en un contexto de cambio. El caso de la ciudad de Valencia", CIRIEC-España, Revista de Economía Pública, Social y Cooperativa, 91, 31-58.

SLIMANE, K.B. Y LAMINE, W. (2017): A transaction-based approach to social innovation, Entrepreneurship and innovation, 18 (4), 231-242.

TORRES, F. Y GARCÍA, P. (2013): La ciudad fragmentada. Análisis comparativo de cuatro barrios emblemáticos, en Cucó i Giner, J. (dir.) (2013): La ciudad pervertida. Una mirada sobre la Valencia global. Anthropos, Barcelona, 191-212 